\title{
CYTOREDUCTIVE SURGERY (CRS) AND HYPERTHERMIC INTRAPERITONEAL CHEMOTHERAPY (HIPEC) IN PATIENTS WITH ADVANCED OVARIAN, FALLOPIAN TUBE OR PRIMARY PERITONEAL CANCER: OUR EXPERIENCE IN 108 PATIENTS
}

\author{
M. E. Căpîlna* B. Moldovan**, C. Rad**, A. A. Moldovan**, A. L. Cozlea*, A. Fandi*, M. \\ Gheorghe*, S. L. Kiss* \\ * - First Obstetrics and Gynecology Clinic, University of Medicine, Pharmacy, Science and \\ Technology, Targu Mures, Romania, Europe \\ ** - St. Constantin Hospital, Brasov, Romania, Europe
}

Objective. Current evidence suggests that complete CRS and HIPEC is a feasible option for patients with advanced ovarian, fallopian or primary peritoneal cancer with potential benefits that may exceed the survival outcomes of current treatment - surgical debulking and intravenous platinum- and taxane-based chemotherapy. Methods: It is a retrospective study including 108 patients with primary or recurrent peritoneal carcinomatosis, operated between June 2013 and March 2019, with a mean age of 53.7 years. Results. Seventyeight patients $(72 \%)$ had primary debulking and 30 (27\%) had surgery for a recurrent disease. The peritoneal cancer index (PCI) was below 15 in 50 patients $(46 \%)$ and above 15 in $58(53 \%)$, respectively. Together with total peritonectomy, large bowel resection was performed in 55 patients $(50.9 \%)$, small bowel resection in 13 $(12 \%)$, and splenectomy in $38(35 \%)$. Other upper abdominal procedures included liver resection $(13 \%)$, colecistectomy $(35 \%)$, gastric resection $(1.8 \%)$, diaphragm resection $(12 \%)$, etc. Microscopically complete cytoreduction (CC0) was achieved for 68 patients (63\%), macroscopic cytoreduction (CC1) for 35 (32\%), and gross tumour debulking (CC2) for 5 $(4 \%)$.
Only 3 patients (2.7\%) have been reoperated. For HIPEC, Cisplatin and respectively, Doxorubicin were both used for 30 patients (27\%). Other regimen included Cisplatin plus Doxorubicin (41\%), Cisplatin plus Mitomicine or Mitomicine alone. Nine patients (8\%) died of disease, 15 (13\%) are alive with reccurent disease, and 84 (77\%) are disease-free, but the follow up is short. Conclusions. HIPEC after extensive CRS for advanced gynecological cancer with peritoneal carcinomatosis is a feasible option with promising results.

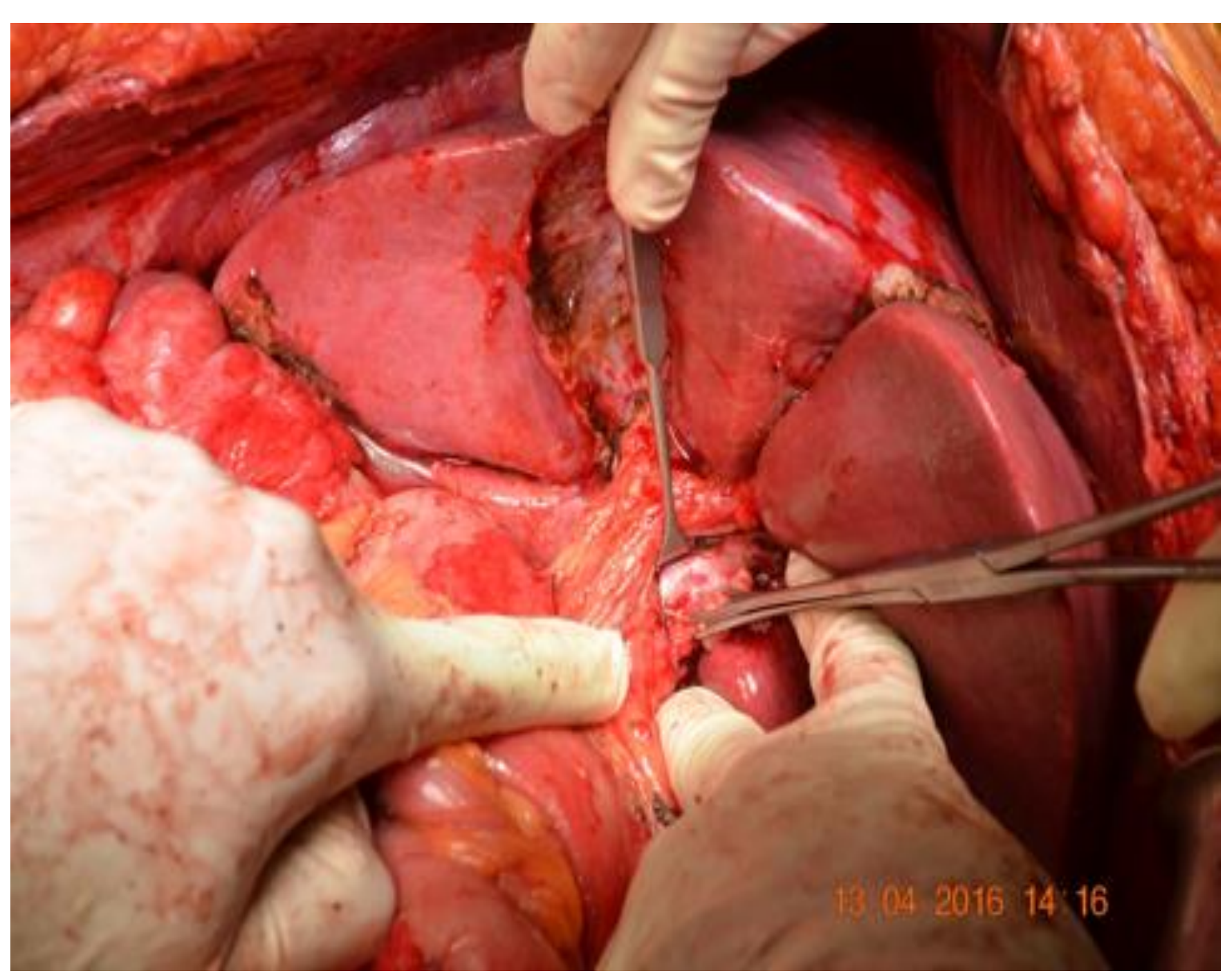

